\title{
Parametric forcing of scroll-wave patterns in three-dimensional excitable media
}

\author{
Rolf-Martin Mantel \\ Institute for Mathematics and Its Applications \\ 514 Vincent Hall \\ University of Minnesota \\ Minneapolis, MN $55455 \quad U S A$ \\ Dwight Barkley \\ Mathematics Institute \\ University of Warwick \\ Coventry $\mathrm{CV}_{4} \mathrm{7AL} \quad \mathrm{UK}$ \\ barkley@maths.warwick.ac.uk
}

\begin{abstract}
The dynamics of axisymmetric and twisted scroll rings under homogeneous periodic forcing is studied numerically. The collapse of axisymmetric rings can be enhanced or retarded significantly with resonant forcing. Twisted scroll rings exhibit the phenomenon of resonant drift in a direction normal to the axis of the central filament. The scaling of mean and fluctuating axial drift due to forcing is found. It is argued that the appropriate symmetry group for the dynamics of the unforced twisted scroll ring is $\mathbb{S E}(2) \times \mathbb{R}$. Ordinary differential equations based on symmetry considerations explain the dynamics without resort to law of filament motion and the local geometry hypothesis.
\end{abstract}

Key words: excitable media, Euclidean symmetry, scroll wave, parametric forcing

\section{Introduction}

The system we consider is a three-dimensional excitable medium, such as a thick layer of the excitable Belousov-Zhabotinsky reagent[1-6]. In such a medium, one finds persistent scroll waves that are organized around onedimensional filaments ranging in complexity from simple rings to complicated links, knots, and tangles[7-12]. These scroll waves are thought to be similar 
in many respects to the waves of electrical activity associated with certain cardiac arrhythmias, and in this way the Belousov-Zhabotinsky system can be considered to be a caricature of cardiac tissue[13]. In this paper we address what happens to scroll waves when weak, homogeneous parametric forcing is applied to the medium and to what extent the resulting dynamics can be understood through low-dimensional dynamical systems analysis without resorting to laws of filament dynamics[14-17] and Winfree's local geometry hypothesis[10,11].

We first recall a few basic facts about homogeneous parametric forcing of a two-dimensional excitable medium in which one finds spiral waves. The problem has been considered extensively experimentally and theoretically[1828]. Fig. 1 summarizes the most notable effect of weak forcing on a rotating spiral wave. In Fig. 1(a) is shown a rotating spiral wave in a standard reactiondiffusion model of excitable media (presented in the next section). The spiral rotates about a fixed center with frequency $\omega_{1}$ and the path of the tip is a circle. Fig. 1(b) shows the linear drift induced by resonant parametric forcing: forcing frequency $\omega_{f}$ equal to $\omega_{1}$. Fig. 1(c) shows the dynamics for forcing near such a resonance. Letting $\Delta=\omega_{1}-\omega_{f}$ denote the detuning between natural spiral frequency and forcing frequency, and $R_{2}$ denote the large secondary radius resulting from the forcing, then in the vicinity of the resonance these are related by $R_{2} \sim 1 / \Delta$, and at resonance $(\Delta=0), R_{2}=\infty$. The drift speed at resonance grows as the amplitude of the forcing (not shown). Note that the curves in Fig. 1(c) are not from reaction-diffusion simulations but from ordinary differential equations obtained from the analysis in [28].

The spiral motion resulting from resonant or near resonant forcing on spiral waves, as illustrated in Fig. 1, has been known for some time and this effect has been proposed as a method for treating certain cardiac arrhythmias by using low-voltage stimuli to remove spiral waves from cardiac tissue[18-20,22,25,26]. Whether or not this idea has any merit in practice, almost nothing is known about how parametric forcing acts in three-dimensional media. Recently analysis has begun of periodic forcing on three-dimensional structures in excitable media using bifurcation theory [29]. However, as far as we are aware, there have been no published numerical or experimental studies of how parametric forcing affects scroll waves. In this paper we study the dynamics of unforced and forced scroll rings. We consider briefly the axisymmetric scroll ring, but focus mainly on the twisted-scroll ring. We provide accurate numerical data for the dynamics and compare with predictions of dynamical systems theory. 


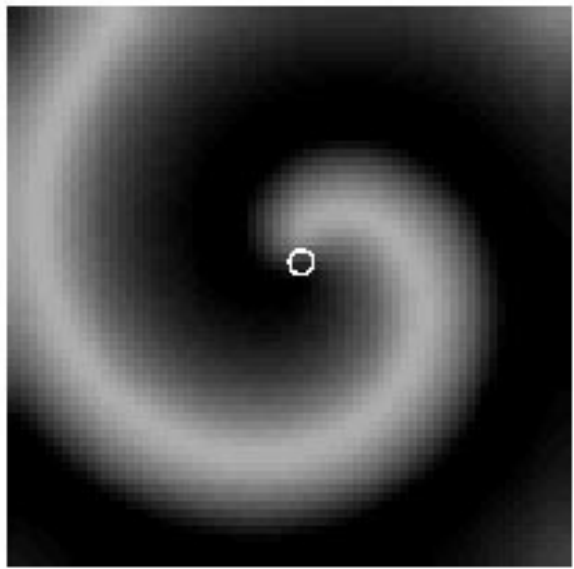

a

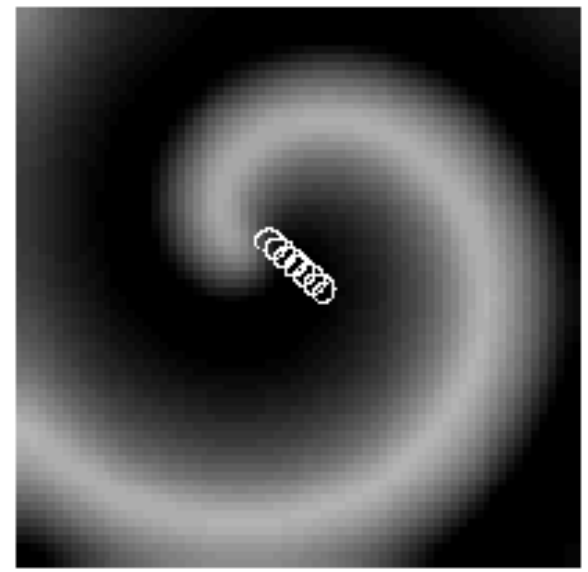

$\mathrm{b}$
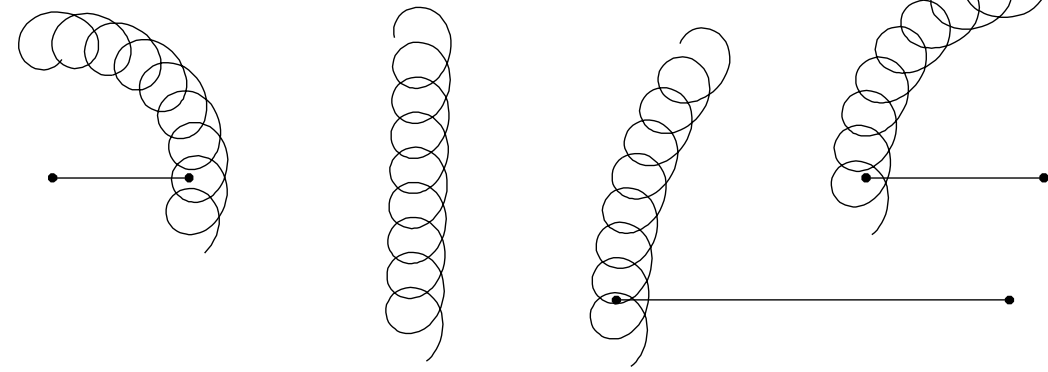

$\frac{1}{\Delta}=9.2$

$\frac{1}{\Delta}=\infty$

$\frac{1}{\Delta}=-25.1$

$\frac{1}{\Delta}=-11.5$

C

Fig. 1. Effects of periodic forcing on spiral-wave dynamics. (a) Periodically rotating solution of the reaction-diffusion model considered in this paper (model parameters are: $a=0.8, b=0.05$, and $\epsilon=0.02$ ). Gray-scale shows values of the slow species and the white curve shows the path of the spiral tip. (b) Conditions as in (a), except with parametric forcing in 1-1 resonance with the spiral rotation frequency, $(b=0.05+0.0035 \sin (1.7434 t))$. (c) The effect of parametric forcing in the vicinity of a 1-1 resonance. $\Delta$ denotes the frequency detuning and line segments show the secondary radius $R_{2}$ of the "tip paths". 


\section{Model and Methods}

For our numerical simulations, we use the two-variable reaction-diffusion equations

$$
\frac{\partial u}{\partial t}=f(u, v)+\nabla^{2} u, \quad \frac{\partial v}{\partial t}=g(u, v)+D_{v} \nabla^{2} v
$$

where the functions $f(u, v)$ and $g(u, v)$ express the local reaction kinetics of the two variables $u$ and $v$. The diffusion coefficient for the $u$ variable is scaled to unity, and thus $D_{v}$ is the ratio of diffusion coefficients. For the reaction kinetics, we use:

$$
\begin{aligned}
& f(u, v)=\frac{1}{\varepsilon} u(1-u)\left(u-u_{\mathrm{th}}(v)\right) \\
& g(u, v)=u-v
\end{aligned}
$$

with $u_{\mathrm{th}}(v)=(v+b) / a$. This choice differs from traditional FitzHugh-Nagumo equations in a way that allows for fast computer simulations[30,31]. We apply periodic forcing by sinusoidally varying the excitability threshold through the parameter $b: b(t)=b_{0}+A \cos \left(\omega_{f} t\right)$. The amplitude $A$ and frequency $\omega_{f}$ (or equivalently period $\left.T_{f}=2 \pi / \omega_{f}\right)$ of the forcing are the parameters varied in this study. We keep the other model parameters fixed at $a=0.8, b_{0}=0.01$, $\varepsilon=0.02$, and $D_{v}=0$. Without forcing, the medium is strongly excitable. In two dimensions, the equations generate rigidly rotating spirals with small cores very similar to that shown in Fig. 1(a). These spirals are far from the meander instability [32] and initial conditions converge quickly to rotating waves.

We use a third-order semi-implicit stepping routine to timestep $f$, combined with explicit Euler timestepping for $g$ and the Laplacian term. In the evaluation of $f$ and in the diffusion of $u$, we take into account that $u \simeq 0$ in a large part of the domain, and that $f(0, v)=0$. We use a 19-point stencil with good numerical properties (isotropic error, mild time-step constraint) for approximating the Laplacian operator. Periodic boundary conditions are imposed in the $z$ direction to allow solutions to move freely in that direction; Neumann boundary conditions are imposed in the other two directions ( $x$ and $y$ directions). We initially simulate with numerical parameters corresponding to moderate resolution: box length $L=36$, grid spacing $h=3 / 7$, time step $\triangle t / \epsilon=2.4$. For high accuracy studies of the twisted scroll ring, we use a higher resolution of $L=24, h=2 / 7, \Delta t / \epsilon=0.6$. Complete numerical details for the three-dimensional simulations are given in [31]. 
We define the scroll filament to be the intersection of the two iso-surfaces $u=1 / 2$ and $v=u_{\mathrm{th}}(1 / 2)$. In two dimensions this defines the spiral tip[30]. For parameter values in this study, two-dimensional spirals trace out small circles (similar to that in Fig. 1). In three dimensions, filaments undergo small-scale circulations at the period of wave rotations (corresponding to rotations in two dimensions) and also slower large-scale motions associated with movement of the scroll structures in three space. We are primarily interested in these slow motions.

Focusing on filaments rather than the full concentration fields reduces the complexity of the structures to be analyzed. However, even this is not sufficient to obtain a low-dimensional description of scroll waves. For the waves we consider here the filaments are or are close to simple geometric objects (lines and circles) and this allows us to reduce the infinite-dimensional dynamics to the dynamics of simple geometric objects described by a few coordinates (centers, radii etc.) and we need only save this small number of quantities during long simulations. For scroll rings, the obvious object to fit is a circle. For axisymmetric rings, the numerically computed filaments are circles to within numerical precision. For twisted rings, the filament is composed of two pieces, one approximately a line and the other approximately a circle. We focus on the nearly circular part of the filament and find the best approximating circle.

We have used the following algorithm for our fitting. First consider finding a best fit sphere to a set of points $\left(x_{i}, y_{i}, z_{i}\right)$ in three dimensions [33]. Defining $Q_{i}=x_{i}^{2}+y_{i}^{2}+z_{i}^{2}$, the following set of equations gives the center $(a, b, c)$ of the sphere:

$$
a\left(\begin{array}{c}
\operatorname{var}(x) \\
\operatorname{covar}(x, y) \\
\operatorname{covar}(x, z)
\end{array}\right)+b\left(\begin{array}{c}
\operatorname{covar}(x, y) \\
\operatorname{var}(y) \\
\operatorname{covar}(y, z)
\end{array}\right)+c\left(\begin{array}{c}
\operatorname{covar}(x, z) \\
\operatorname{covar}(y, z) \\
\operatorname{var}(z)
\end{array}\right)=\frac{1}{2}\left(\begin{array}{c}
\operatorname{covar}(x, Q) \\
\operatorname{covar}(y, Q) \\
\operatorname{covar}(z, Q)
\end{array}\right),(3)
$$

where var and covar refer to variance and covariance of the data points. The radius then is calculated as

$$
r=\sqrt{a^{2}+b^{2}+c^{2}+\bar{Q}-2 a \bar{x}-2 b \bar{y}-2 c \bar{z}}
$$

where $\bar{x}=$ mean(x), etc. Eq. (3) become nearly singular if the points are nearly coplanar as in the case of a scroll ring. The size of the eigenvalue closest to zero gives a measure of how close the points are to a plane, and the associated normalized eigenvector gives a unit normal for a family of planes. Replacing the nearly singular direction with the equation of the plane going through the mean position $(\bar{x}, \bar{y}, \bar{z})$ allows us to calculate the center and radius of the best fit circle, which we denote $(X, Y, Z)$ and $R$, respectively. In this way full concentration fields are projected onto four scalars. In addition, we output the unit normal vector and the error in the fit. The later is used to assess the 


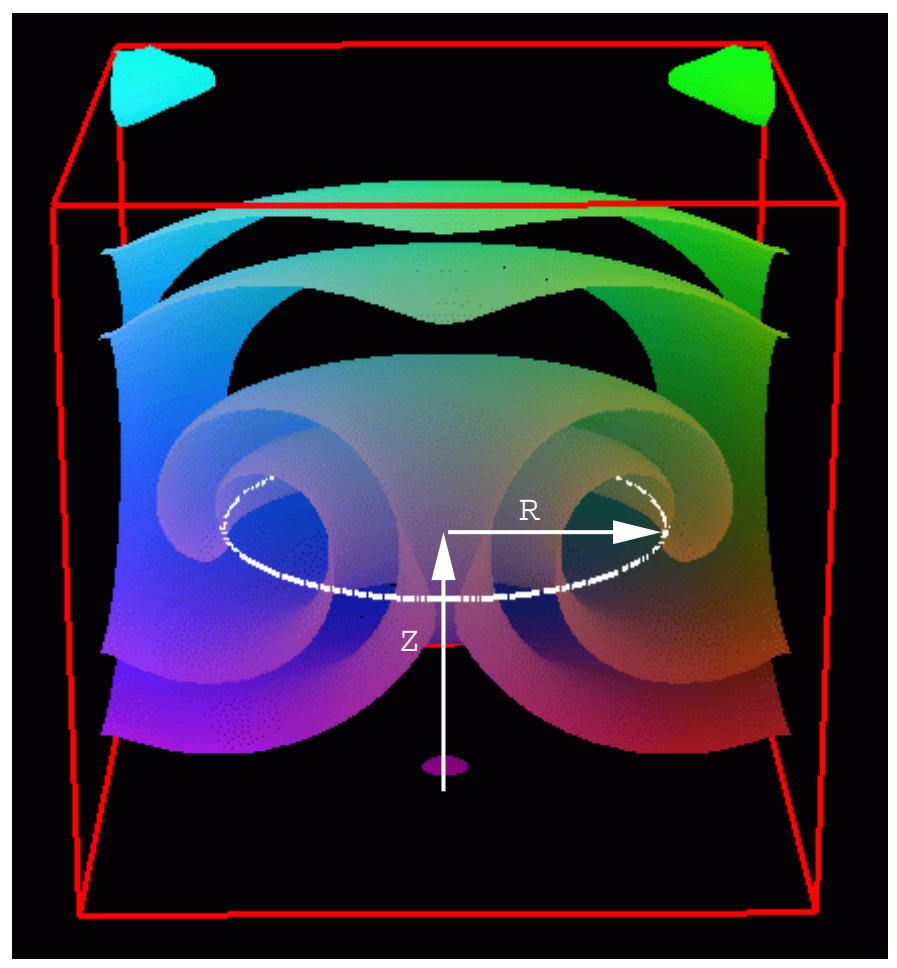

Fig. 2. Axisymmetric scroll ring. Shown is the iso-surface $u=1 / 2$ (clipped halfway through the volume). The filament (white) forms a planar ring parameterized by the radius $R$ and location $Z$ along the symmetry axis. Model parameters are as given in Sec. 2. The domain has sides of length $L=40$.

validity of fitting a circle to the approximately circular filament of the twisted scroll ring in Section 3.

\section{Results}

\subsection{Axisymmetric scroll rings}

A scroll ring is an axisymmetric solution of the reaction-diffusion equations which contains a spiral pattern in each azimuthal slice; see Fig. 2. The filament formed from the "tips" of these spirals is a planar ring. We take the axis of symmetry to be the $z$ axis. The dynamics of scroll rings are necessarily limited to changes in radius, $R$, and position, $Z$, along the axis of symmetry. In a singly diffusive medium $\left(D_{v}=0\right)$ it is well established that an axisymmetric ring drifts and shrinks at a speed inversely proportional to its radius (e.g. $[34,3,10,12,35])$.

Under parametric forcing of the medium, there are two possibilities for the behavior: either the axisymmetry of the solution breaks, in which case one could 

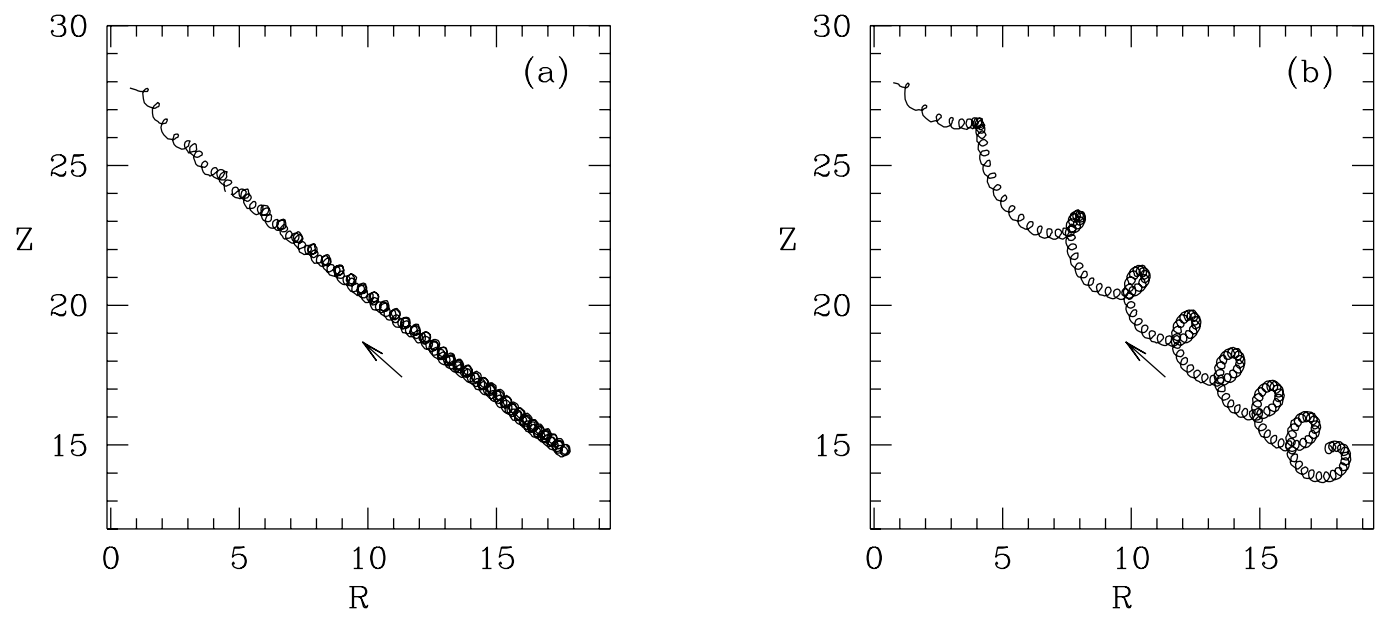

Fig. 3. Periodic forcing of a scroll ring. Plotted is the path of a scroll ring in $R-Z$ coordinates for (a) forcing away from resonance $\left(T_{f}=4.2\right)$ and (b) near resonant forcing $\left(T_{f}=3.5\right)$. The period of the unforced ring is not constant but $T_{1} \approx 3.3$.

expect the scroll filament to become non-planar and possibly quite complicated, or else the axisymmetry does not break, in which case the dynamics of the forced scroll ring can still be captured by measurements of $R$ and $Z$. In practice we have limited ourselves to weak periodic forcing and have found no evidence of symmetry breaking under these conditions.

For our numerical simulations, we start with an initial condition created by the complex polynomial $p\left(z_{1}, z_{2}\right)=z_{2}$ (see Appendix A). We then simulate for two to three rotations of the scroll in the absence of forcing to enable the scroll ring to settle into a state with approximately constant period. We take this state as an initial condition for our forcing experiments. The radius $R$ and center $(X, Y, Z)$ are obtained via the fitting procedure described in Section 2. The $(X, Y)$ values of the center are found to be constant as expected for the axisymmetric ring. Note that while we simulate scroll rings in cubical domains, the solutions are axisymmetric to a high degree of precision. This is similar to the situation in two dimensions in which spiral waves behave as though in an infinite medium so long as the spiral centers are more than a wavelength from domain boundaries[32,36].

Fig. 3 shows filament paths for weak periodic forcing of a scroll ring in two cases, one near frequency resonance and the other away from resonance. Away from resonance, the scroll ring collapses in almost the same way as in the absence of forcing: the path in $R-Z$ coordinates is straight (averaged over the small-scale circular motions) until $R$ becomes very small. The only difference between this and the unforced case is that the small-scale circular motions associated with the scroll wave rotations are slightly altered under forcing. 


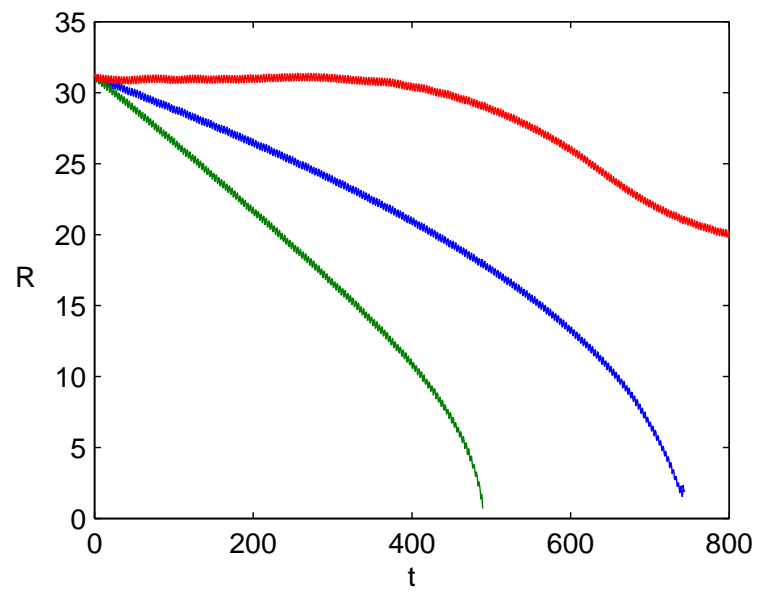

Fig. 4. Periodic forcing of a scroll ring. Plotted is $R$ versus $t$ for resonant periodic forcing $\left(T_{f}=3.31, A=0.00125\right)$. Shown is no forcing (middle curve), forcing at a phase that gives most rapid collapse, and forcing at a phase that give maximum stabilization. Collapse of a scroll ring is delayed for approximately 100 wave rotations.

Close to resonance, however, there is a sizeble deviation from straight-line collapse in $R-Z$ coordinates. Recall the effect of parametric forcing on a spiral wave. As a frequency resonance is approached, the secondary radius of the quasiperiodic tip path grows as $R_{2} \sim 1 / \Delta$, where $\Delta \equiv \omega_{1}-\omega_{f}$. Thus the near-resonant motion of the scroll ring can be understood simply as the large-scale quasiperiodic motion of a forced spiral wave (in $R-Z$ coordinates) superimposed on the natural shrink and drift dynamics. Note that the shrink and drift rates are themselves not constant but proportional to $1 / R$. Thus as as $R \rightarrow 0$, the shrink and drift speeds increase, whereas the bi-periodic motions due to forcing do not. This accounts for the uncoiling of the filament path as $R \rightarrow 0$. There is also a small shift in the rotation frequency of the scroll as $R \rightarrow 0$ but this seems to have little effect on the dynamics.

The fact that resonant forcing of a spiral wave induces a linear drift leads to the conjecture that resonant forcing at the appropriate amplitude and phase could be used to stabilize a collapsing scroll ring. That is, if the forcing-induced drift can be adjusted to counteract the shrink, then the radius of the scroll ring can be stabilized, though drift along the axis of symmetry would still occur. Likewise, for the same amplitude and frequency, but another choice of phase for the forcing it should be possible to greatly accelerate the ring collapse or to enhance the drift along the symmetry axis.

We have numerically tested this hypothesis and the results are shown in Fig. 4. Collapse of the scroll ring can be significantly accelerated or delayed with quite samll forcing. With effort we have succeeded in stabilizing the scroll ring at a constant radius $R$ for more than 100 wave rotations (over 300 time units). Thereafter the ring begins to collapse. Thus we found that stabilization of the 

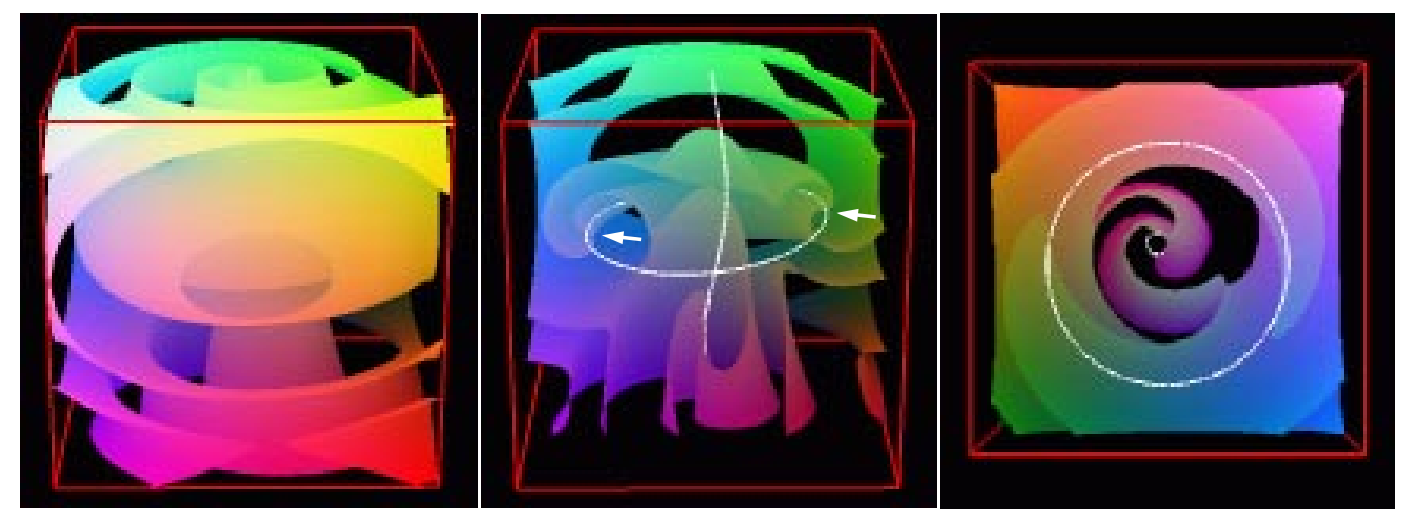

Fig. 5. Twisted scroll ring. Iso-surface $u=1 / 2$ (left), clipped iso-surface with filament (middle), and clipped iso-surface and filament from above (right). Arrows emphasize the change in "spiral phase" in rotating around the filament (cf. figure 2). Note, these images illustrate the structure of fields for the twisted ring, but this is not the asymptotic state. The domain has sides of length $L=40$.

ring with fixed-frequency forcing is probably possible but difficult in practice because the resonance condition must be satisfied to a very high degree. The problem is compounded by the fact that once forcing is applied, the rotation frequency of the scroll frequency changes slightly. It should be possible to completely stabilize a scroll ring either with further adjustments of the forcing period and phase or by applying feedback control to the forcing.

In principle one could attempt a low-dimensional dynamical systems description of the forced scroll ring (similar to the description considered in the following section of the twisted scroll ring). Ashwin and Melbourne [37] have done this by considering the scroll ring to be a stable state of the system. However, as axisymmetric scroll rings are nearly always, in fact, transient structures we have not pursued a low-dimensional description of these states.

\subsection{Twisted scroll rings}

A twisted scroll ring is a solution to the reaction-diffusion equations with an approximately circular filament in which the phase of the "spiral" is not the same at each azimuthal location. Instead the spiral phase changes around the ring by a multiple of $2 \pi$. We consider the simplest case of a once twisted scroll ring (Fig. 5) for which the phase change is $2 \pi$. Topologically there must be another filament passing through the center of the filament ring [7], see also[10]. Thus even though one generally refers to the state as a twisted scroll ring, the filament consists of two pieces.

An initial condition for a twisted scroll ring is generated by the polynomial $p\left(z_{1}, z_{2}\right)=z_{1} z_{2}$ (see Appendix A). The straight part of the filament is then 

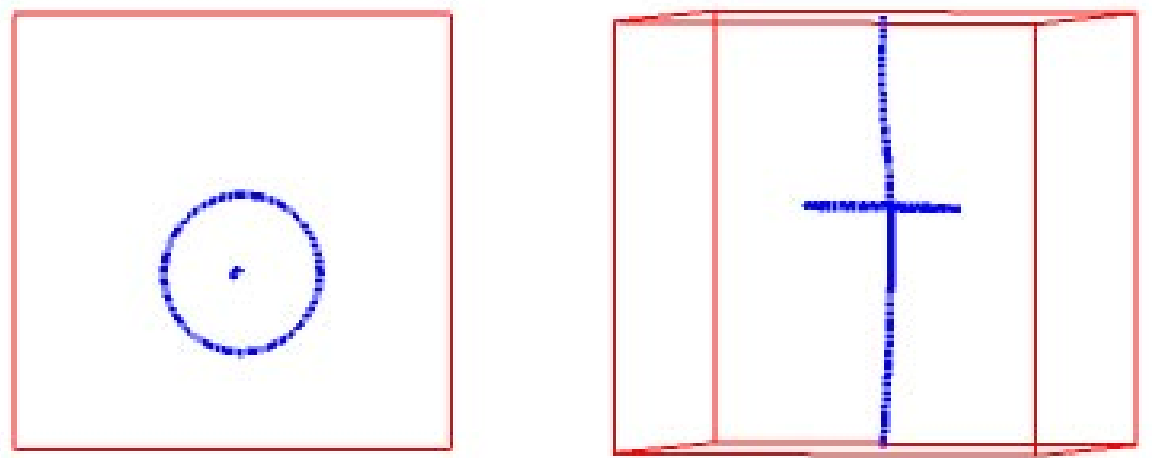

Fig. 6. Two views of the filament for the asymptotic twisted scroll ring. Top view showing projection onto $x-y$ coordinates (left) and side view with the $z$-axis vertical (right). The ring is nearly planar and the central filament is nearly straight and parallel to the $z$-axis. The domain has sides of length $L=24$, that used for most simulations.

parallel to the $z$ axis (the periodic direction in our simulations). Starting from the polynomial initial conditions, we simulate the reaction-diffusion equations long enough for the system to reach an asymptotic state. During the transience, the ring shrinks until the interaction between the ring and the central filament stops the contraction. This final asymptotic state provides the initial condition used for our forcing experiments. The state in Fig. 5 is not this final asymptotic state, but we choose to show this early-time state because the structure of the fields is clearly seen. Fig. 6 shows the filaments for the asymptotic state. It can be seen from the top view that the ring is indeed very close to circular and that the central filament is close to straight. Before describing the forcing studies, it is necessary to consider in some detail the dynamical properties of the unforced state.

\subsubsection{Dynamics of the unforced twisted scroll ring}

The important issue from our point of view is what symmetry group is appropriate for describing the dynamics of the state, for this will dictate a lowdimensional description of the forced dynamics. Unlike the untwisted scroll ring, the twisted scroll ring has no spatial symmetries (apart from the artificial periodic symmetry in our numerical treatment) and this is why the ring is not exactly a circle. However, the twisted scroll ring has a space-time symmetry: under time evolution the wave uniformly rotates and drifts according to

$$
\mathbf{u}(t)=T_{c_{1} t} R_{\omega_{1} t} \mathbf{u}(0),
$$


where $\mathbf{u}=(u, v), R_{\gamma}$ is a rotation through angle $\gamma$ about some fixed axis, and $T_{d}$ is a translation of distance $d$ in that same direction. Hence $\omega_{1}$ is the rotation frequency and $c_{1}$ is the drift speed. In our case the rotation axis is parallel to the $z$-axis so the drift is also in the $z$-direction.

To verify this, we have simulated the twisted ring for hundreds of wave rotations and have measured the drift and rotation of the solution. We then applied the inverse transformations to the time dependent solution: $T_{-c_{1} t} R_{-\omega_{1} t} \mathbf{u}(t)$. The resulting state was found to be steady, i.e. the twisted scroll ring is a relative equilibrium of the system. We have verified that to a high degree of precision the axis of rotation and direction of translation are parallel to the $z$ axis.

It is very significant that the rotation and translation axes align with the $z$ axis. It has been shown that in autonomous systems with three-dimensional Euclidean symmetry $\mathbb{E}(3)$, the evolution of a non-symmetric relative equilibria is generically rotation about an axis together with translation along the same axis [37-39]. This is as we have found. However, the symmetry analysis indicates that the axis points in an arbitrary direction. This is reasonable because a state with no spatial symmetry should drift in an arbitrary direction for there is nothing to determine a specific direction. However, this is not in agreement with our observation that the motion has a preferred direction: the $z$-axis. Of course in our simulations, we impose periodic boundary conditions in the $z$-direction. We conjecture, however, that even in an infinite medium the axis of rotation and drift would still align with the central filament.

Our argument is as follows. First, even in an infinite domain a twisted scroll ring has no spatial symmetry, so it is not a spatial symmetry that selects an axis. What selects the axis is the direction along which the central filament extends to infinity, which without loss of generality can be taken to be the $z$-direction. In each two-dimensional slice normal to the $z$ axis one finds a spiral pattern away from the axis. This can be seen to some extent in Fig. 5. As with usual two-dimensional spiral waves, these spirals behave nicely under rotations (around the $z$-axis) because small rotations result in small changes in the fields, even arbitrarily far from the axis. However, small rotations around any axis not parallel to the $z$ axis produce arbitrarily large changes in the fields, i.e. rotations act discontinuously on the state. Consider for example a small rotation around the $x$-axis. This would rotate the direction of the central filament and thus move the filament through a distance that would become unbounded as $z \rightarrow \infty$. Thus the evolution of the state will not include such rotations and so they should be excluded from a description of the dynamics. Thus a model description will include only the two-dimensional Euclidean symmetry in the plane perpendicular to the filament and the one dimensional Euclidean symmetry along the filament axis. For describing the dynamics of a single twisted scroll ring one would not need to include reflections in the plane 

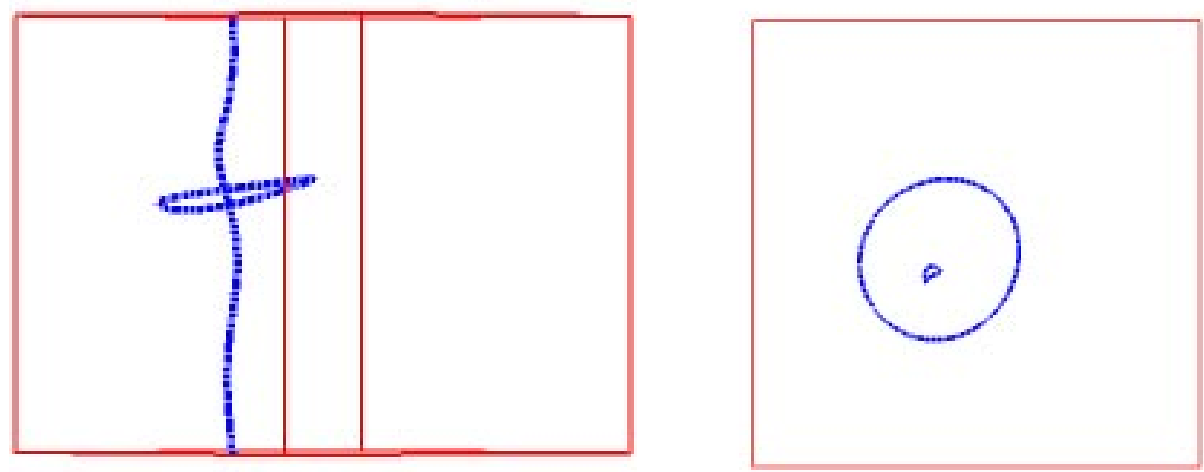

Fig. 7. Filaments of twisted scroll ring with parametric forcing. Forcing is at resonance $T_{f}=T_{1}=2.9925$ with amplitude $A=0.002$. There is considerable change in shape from the unforced case.

and reflection along the filament axis, though we find it natural to model these also. We address this further in Section 3.2.3. We thus conjecture that the relevant symmetry group for the dynamics of a single twisted scroll ring is $\mathbb{S E}(2) \times \mathbb{R}$. This is consistent with our numerical experiments which are themselves representative both of other simulations and of what would occur experimentally.

A different issue concerning the unforced twisted ring is that there is bistability between two nearly identical states, at least in our simulations with finite periodicity in $z$. If one starts a simulation from the polynomial initial condition, then the asymptotic state reached is not the state shown in Fig. 6, but a very slightly different one. If however, one perturbs that state, via periodic forcing for example, and then removes the perturbation, the state relaxes to that shown in Fig. 6. The two states are virtually indistinguishable by eye. The drift speed and rotation frequencies for the two states are very close, but measurably different (about 1 part in $10^{4}$ ). For determining the scaling behavior of the system as the forcing amplitude, $A$, goes to zero, it is necessary to use the state in Fig. 6 as the $A=0$ limit. It is possible that the existence of the two, nearly identical states is a finite-size effect due to the fact that the periodicity in $z$ constrains the twist in the central filament, and that in an infinite domain the two states would be the same.

\subsubsection{Periodic forcing of a twisted scroll ring}

We now turn to the effect of periodic forcing on the twisted scroll ring. In this section we present our numerical results and in section 3.2.3 we discuss the interpretation in terms of dynamical systems theory. Qualitatively, forcing 

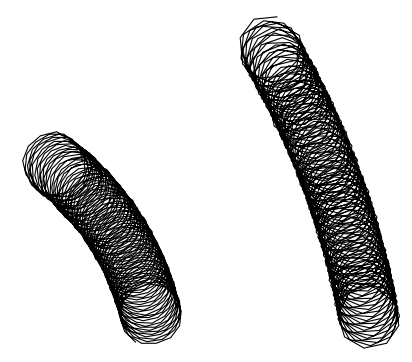

$\mathrm{T}=2.985$

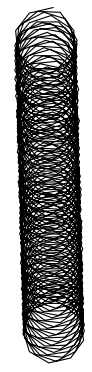

$\mathrm{T}=2.9895$

$\mathrm{T}=2.988$
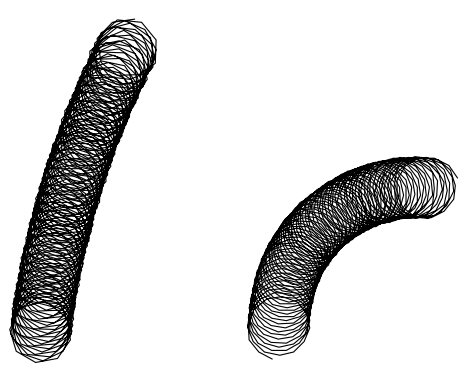

$\mathrm{T}=3$

$\mathrm{T}=2.991$

Fig. 8. The effect of near-resonant and resonant periodic forcing on the twisted scroll ring as seen projected onto the $X-Y$ plane. Five values of the forcing period $T_{f}$ are shown at fixed forcing amplitude $A=0.0005$.

does not change the wave fronts much away from the filament. Forcing can introduce significant changes in the shape of the filament, however (Fig. 7). The central filament is noticeably helical. The ring is distinctly oval, not centered around the central filament, and not confined to a plane. Despite this the ring remains, in some sense, perpendicular to the filament where it passes through the ring's "center". While the ring is not circular, fitting to a circle is still a valid method for projecting onto a small number of variables: $(X, Y, Z, R)$. In any case, at this forcing amplitude, the largest we consider, the only measurement we use is the drift in the $Z$-coordinate and the fit is sufficient for this purpose.

The interesting dynamical aspects of the forced scroll ring along the filament axis, $Z$ coordinate, and perpendicular to the axis, $(X, Y)$ coordinates, are independent and thus we consider these separately. The important behavior in the $X-Y$ dynamics is resonant drift. That is, if the twisted scroll ring is forced at resonance, then drift occurs in $X-Y$ exactly as for spiral waves. This is shown in Fig. 8 where we plot $X-Y$ paths for five forcing frequencies at fixed forcing amplitude. The similarity to the dynamics of spiral waves in Fig. 1 is apparent. (Note that in the absence of forcing the dynamics in the $X-Y$ plane is a rotating wave, i.e. the center of the best-fit circle traces out a circular path.)

Typical drift dynamics in the $Z$ coordinate under periodic forcing is shown in Fig. 9. Not surprisingly, the instantaneous drift speed varies at the forcing period. What is at issue here is how the drift scales with forcing amplitude. To quantify this we define the instantaneous drift speed $c(t)=\mathrm{d} Z / \mathrm{d} t$, which can then be separated into mean and fluctuating parts $c(t)=c_{1}+C(t)$ where $C(t)$ is periodic with zero mean over the forcing period: $<C(t)>_{T_{f}}=0$. 

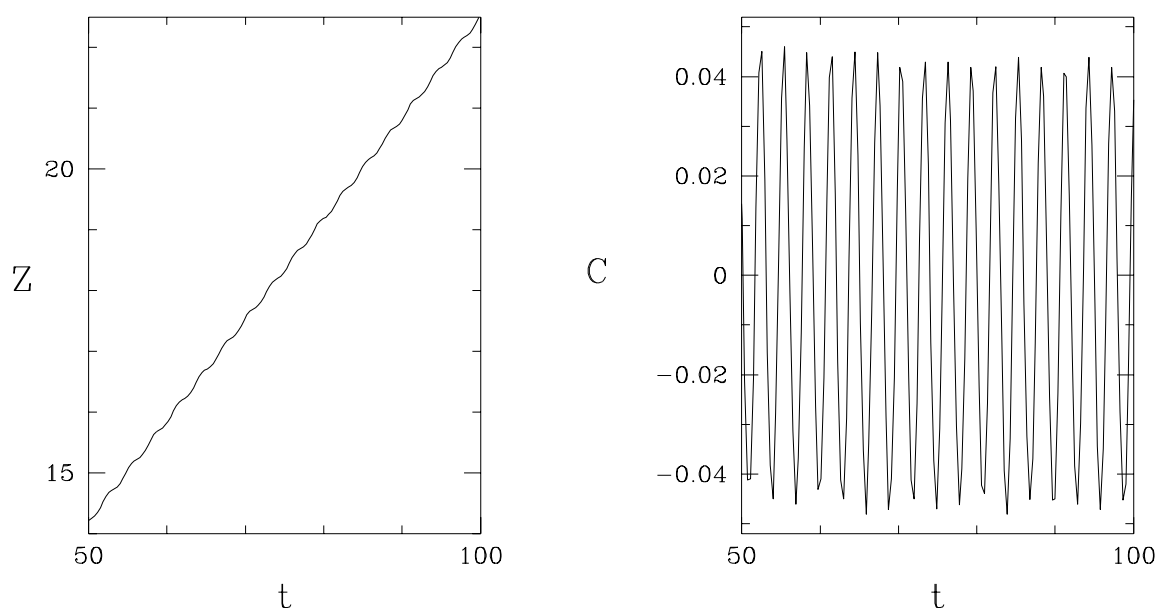

Fig. 9. Axial drift dynamics, $Z(t)$, under parametric forcing. Parameter values are $A=0.001$ and $T_{f}=2.9895$.
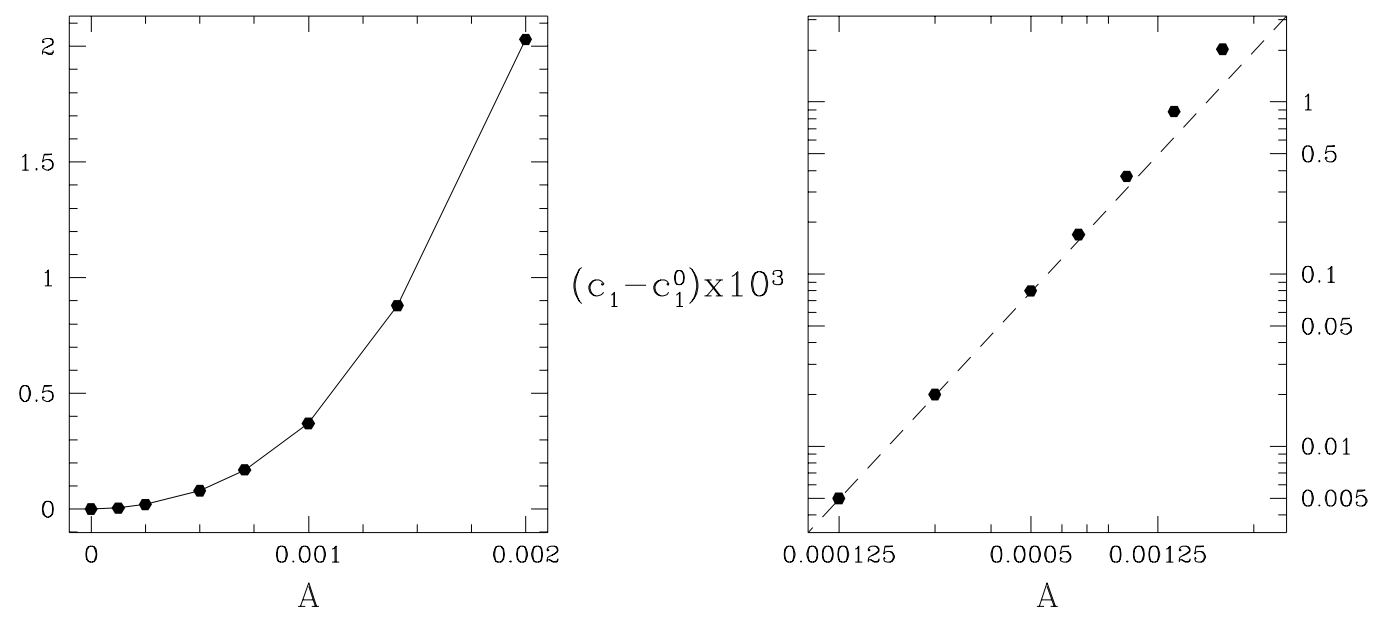

Fig. 10. Plots showing the dependence of mean drift speed $c_{1}$ on forcing amplitude, where $c_{1}^{0}=0.165705$ is the drift speed without forcing. The dashed line has slope 2. The induced change is quadratic in the forcing amplitude.

In Fig. 10 we show the modification of the mean drift induced by forcing by plotting $c_{1}-c_{1}^{0}$ as a function of forcing amplitude, where $c_{1}^{0}$ is the drift without forcing. The mean drift speed is seen to scale as $A^{2}$ for small forcing amplitude. Fig. 11 shows $|C|$, the maximum of $C(t)$, as a function of forcing amplitude. Here it is seen that the magnitude of the fluctuating drift is proportional to forcing amplitude for small forcing. In summary, we find the following scalings as the forcing amplitude goes to zero: $c_{1}-c_{1}^{0}=O\left(A^{2}\right)$ and $C(t)=O(A)$.

\subsubsection{Dynamical systems description}

Assuming that the symmetry group for the dynamics of the twisted scroll ring is $\mathbb{S E}(2) \times \mathbb{R}$, one can qualitatively reproduce the dynamics of these scrolls 


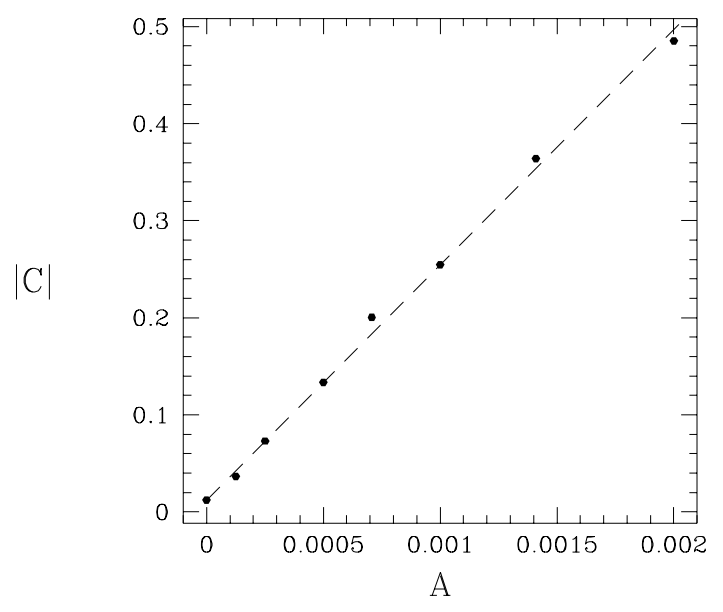

Fig. 11. Amplitude of the fluctuating drift as a function of forcing amplitude. Plotted is the maximum of the fluctuating drift $|C|$ normalized by the mean drift speed $c_{1}$. In the unforced case the drift speed varies by approximately $\pm 1 \%$ due to numerical inaccuracies while at a forcing amplitude of $A=0.002$ the drift speed varies by nearly $\pm 50 \%$.

with ordinary differential equations (ODEs) that have this symmetry. While Fiedler et al. $[38,39,29]$ have developed a general rigorous approach to the problem, they do not include reflectional symmetries in their treatment (the reason is that reflection symmetry is already broken by the twisted scroll ring and so it does not enter into any bifurcation from the twisted scroll state). We take a slightly more global view and consider a set of equations which includes reflections along the filament axis and reflections in the plane perpendicular to the filament; that is we include all the symmetries of $\mathbb{E}(2) \times \mathbb{E}(1)$. (We explain the reason for this later.) The following ODEs model the dynamics of scroll rings:

$$
\begin{aligned}
& \dot{P}=s(\nu) \mathrm{e}^{\mathrm{i} \phi}, \\
& \dot{Z}=c(\nu), \\
& \dot{\phi}=\omega, \\
& \dot{\nu}=\nu \cdot f\left(\nu^{2}, \omega^{2}\right)=\alpha_{0}+\alpha_{1} \nu^{2}+\alpha_{2} \omega^{2}, \\
& \dot{\omega}=\omega \cdot g\left(\nu^{2}, \omega^{2}\right)=\beta_{0}+\beta_{1} \nu^{2}+\beta_{2} \omega^{2},
\end{aligned}
$$

where $P$ is complex, $P=X+\mathrm{i} Y$, and the other variables are real. The functions $s$ and $c$ satisfy $s(\nu)=s(-\nu) \geq 0$ and $c(-\nu)=-c(\nu)$. The exact form of the polynomial functions $f$ and $g$ is not important. Note that in this section $f$ and $g$ will denote the above functions in (5) and not the kinetics functions in Eqs. (2); this should not leed to any confusion.

Eqs. (5) are invariant under the following transformations: 


$$
\begin{aligned}
& R_{\gamma} \cdot\left(\begin{array}{c}
P \\
Z \\
\phi \\
\nu \\
w
\end{array}\right)=\left(\begin{array}{c}
\mathrm{e}^{\mathrm{i} \gamma} P \\
Z \\
\phi+\gamma \\
\nu \\
w
\end{array}\right), \quad \kappa \cdot\left(\begin{array}{c}
P \\
Z \\
\phi \\
\nu \\
w
\end{array}\right)=\left(\begin{array}{c}
P^{*} \\
Z \\
-\phi \\
\nu \\
-w
\end{array}\right) \\
& T_{a b c} \cdot\left(\begin{array}{c}
P \\
Z \\
\phi \\
\nu \\
w
\end{array}\right)=\left(\begin{array}{c}
P+a+\mathrm{i} b \\
Z+c \\
\phi \\
\nu \\
w
\end{array}\right), \quad \kappa_{z} \cdot\left(\begin{array}{c}
P \\
Z \\
\phi \\
\nu \\
w
\end{array}\right)=\left(\begin{array}{c}
P \\
-Z \\
\phi \\
-\nu \\
w
\end{array}\right) \text {, }
\end{aligned}
$$

where $*$ denotes complex conjugation, $R_{\gamma}$ is rotation by angle $\gamma$ about the vertical axis, $\kappa$ is reflection in the $X-Y$ plane (specifically $Y \rightarrow-Y$ ), $T_{a b c}$ is translation of $(X, Y, Z)$ by $(a, b, c)$, and $\kappa_{z}$ in reflection in $Z$. These are the transformations of the group $\mathbb{E}(2) \times \mathbb{E}(1)$.

The interpretation of the equations is as follows. The triple $(X, Y, Z)$ in the ODE model corresponds to a projection of twisted scroll solutions into $\mathbb{R}^{3}$. In particular, it corresponds to the position in physical space of essentially any well-defined, well-behaved point of the solution. We have in mind the center of the best-fit circle used numerically to project the state of the system into $\mathbb{R}^{3}$, and hence we use the same notation in the ODE model (this should not cause any confusion).

It can be seen that the $\dot{\nu}$ and $\dot{\omega}$ equations decouple in system (5) and that $\phi(t), P(t)$, and $Z(t)$ can be found by quadrature once $\nu(t)$ and $\omega(t)$ are known. Note that for given $\nu(t)$ and $\omega(t), P(t)$ and $Z(t)$ are determined independently, i.e. $P(t)$ does not depend on $Z(t)$ and vice versa.

A non-symmetric relative equilibrium occurs at nonzero values $\nu_{1}$ and $\omega_{1}$ for which $f\left(\nu_{1}^{2}, \omega_{1}^{2}\right)=g\left(\nu_{1}^{2}, \omega_{1}^{2}\right)=0$. This corresponds to a state that rotates in $X-Y$ coordinates and drifts in $Z$. To see this one integrates the $\dot{\phi}$-equation to obtain: $\phi(t)=\omega_{1} t+\phi_{0}$. Substituting this into the equation for $\dot{P}$ gives:

$$
\dot{P}(t)=s\left(\nu_{1}\right) \mathrm{e}^{\mathrm{i}\left(\omega_{1} t+\phi_{0}\right)}
$$

which integrates to:

$$
P(t)=P_{0}+R_{1} \mathrm{e}^{\mathrm{i}\left(\omega_{1} t+\phi_{0}+\delta_{1}\right)}
$$


where $R_{1} \equiv\left|s\left(\nu_{1}\right) / \omega_{1}\right|$, and $\delta_{1}=\mp \pi / 2$ depending on the sign of $\omega_{1}$. The dynamics in $Z$ are obtained by integrating the $\dot{Z}$-equation to obtain: $Z(t)=$ $c_{1} t+Z_{0}$, where $c_{1}=c\left(\nu_{1}\right)$ and $Z_{0}=Z(0)$. Thus the state drifts at speed $c_{1}$, where generically $c_{1} \neq 0$. This is as in the analysis of the helix in [29].

We return to the connection between the states in ODEs (5) and scroll waves in the reaction-diffusion system (1). The relative equilibrium given by $\left(\nu_{1}, \omega_{1}\right)$ corresponds to a twisted scroll ring whose central filament is parallel to the $z$ axis, that rotates with frequency $\omega_{1}$ about that axis and drifts with speed $c_{1}$ along that axis. In the reaction-diffusion system there is a family of such states obtain by translations in three space combined with rotations about the $z$ axis. In the ODE system this family is captured by the arbitrary constants $P_{0}, \phi_{0}$, and $Z_{0}$. In our numerical simulations we impose Neumann boundary conditions in the $x-y$ directions and this means that the system in not truly symmetric with respect to $x-y$ translations and rotations about the vertical axis. However, in practice the effect of the boundaries is extremely weak as long as filaments do not get too close to the boundaries. This is exactly as for spiral waves and this is why translational symmetries is an appropriate modeling assumption for these waves $[32,36]$.

If we consider all twisted scroll rings in the reaction-diffusion equations with filaments aligned with the vertical, then there are in fact four families of states related by the two independent reflection symmetries. One may reflect the system in $z$, or in the $x-y$ plane, or both and thereby obtain other solutions. Reflecting in $z$ gives a state that drifts in the opposite direction from the original, but rotates in the same direction. Reflecting in the $x-y$ plane gives a state that drifts in the same direction but rotates in the opposite direction, and finally reflections in both directions give a state that drifts and rotates in the opposite direction from the original. In the ODE model, this is captured by the existence of four symmetrically related states: for each relative equilibrium $\left(\nu_{1}, \omega_{1}\right)$ there are also relative equilibria $\left(-\nu_{1}, \omega_{1}\right),\left(\nu_{1},-\omega_{1}\right)$, and $\left(-\nu_{1},-\omega_{1}\right)$.

The disconnected states are not described if the reflection symmetries are not included into the model. We believe that including reflections in the model ODEs provides a more natural set of equations for modeling the twisted scroll ring observed numerically because the reaction-diffusion equations themselves have reflection symmetries, i.e. both directions of rotation and both directions of drift are observed numerically depending on the initial conditions. The equations we propose would presumably be those obtained rigorously by considering a state symmetric under both reflections $\kappa$ and $\kappa_{z}$ that undergoes simultaneous symmetry breaking bifurcations. The unfolding of such a codimension-two bifurcation would then naturally contain both signs of rotation and both directions of drift. While there is no evidence, as far as we are aware, that such a bifurcation occurs, it is reasonable to postulate such a bifurcation point and describe the numerically observed states as those found 
in its unfolding. In any case it is a simple matter to eliminate the reflection symmetries from the ODE system. One simply considers the system in the vicinity of one of the four symmetrically related states, $\left(\nu_{1}, \omega_{1}\right)$ say. These can then be taken to be given constants and the last two equations in (5) would be dropped; the reflections $\kappa$ and $\kappa_{z}$ would be dropped from (6).

We now turn to the description of periodic forcing. This follows almost exactly the treatment in [31] so we shall not include all the details. In fact, we have already seen in the ODE system that the $P$, i.e. $X-Y$, dynamics does not depend on the $Z$ dynamics. Moreover, Eq. (5) for $P$ is as for two-dimensional spiral waves. It follows immediately that the dynamics of the forced scroll ring projected onto the $X-Y$ plane will be exactly that of a forced spiral wave in two dimensions. Hence the resonant drift dynamics of the scroll ring in Fig. 8 looks just like the resonant drift in Fig. 1 in ODEs describing spiral waves.

The essence of the resonant drift is as follows. With periodic forcing at frequency $\omega_{f}$, the equation for $\dot{P}$ will contain terms, in addition to those in (7), of the form:

$$
\mathrm{e}^{\mathrm{i}\left(\omega_{1}+k \omega_{f}\right) t}
$$

for all integer $k$. Any term of this form integrates to a term of the same form so long as $\omega_{1}+k \omega_{f} \neq 0$. At a resonance, where $\omega_{1}+k \omega_{f}=0$, integration gives a term in $P(t)$ that is linear in $t$ : this is the resonant drift.

For the $Z$-dynamics, we need to consider the form of $c(t) \equiv c(\nu(t))$ when weak parametric forcing at frequency $\omega_{f}$ is applied to the equations. Let the amplitude of the forcing be denoted $\epsilon$ and let $c_{1}^{0}$ be $c\left(\nu_{1}\right)$ in the absence of forcing $(\epsilon=0)$. Then under the assumption that $c$ is smooth and $d c / d \nu \neq 0$ at the unforced state $\nu_{1}$, then $c(t)$ will be expressible in the form:

$$
c(t)=c_{1}^{0}+\sum_{k=-\infty}^{\infty} \hat{c}_{k} \mathrm{e}^{\mathrm{i} k \omega_{f} t}=\left(c_{1}^{0}+\hat{c}_{0}\right)+\sum_{k \neq 0} \hat{c}_{k} \mathrm{e}^{\mathrm{i} k \omega_{f} t}=c_{1}+\tilde{c}(t)
$$

where we have divided $c(t)$ into its mean and fluctuating parts: $c_{1}$ and $\tilde{c}(t)$ respectively.

In the weakly nonlinear regime, the coefficients $\hat{c}_{k}$ will generically scale as: $\left|\hat{c}_{k}\right|=O\left(\epsilon^{|k|}\right)$ for $k \neq 0$. Assuming the forcing has zero mean (as for the sinusoidal forcing we consider), then nonlinearity will shift the mean by $\hat{c}_{0}=$ $O\left(\epsilon^{2}\right)$.

Integrating the $\dot{Z}$ equation, we have:

$$
Z(t)=c_{1} t+C(t)
$$


where

$$
C(t) \equiv \int_{0}^{t} \tilde{c}\left(t^{\prime}\right) \mathrm{d} t^{\prime}=O(\epsilon)
$$

Thus we recover the scalings found numerically in the reaction-diffusion simulations, namely that the mean drift speed scales as $c_{1}=c_{1}^{0}+O\left(\epsilon^{2}\right)$ and the fluctuating drift scales as $C=O(\epsilon)$ in the limit of small forcing.

\section{Conclusion}

We have accurately simulated two types of three-dimensional scroll structures in a reaction-diffusion model of excitable media with parametric forcing. Our particular focus has been on understanding the behavior of these states from a dynamical systems viewpoint. For this we have projected the concentration fields of the reaction-diffusion equations into a four-dimensional space by computing scroll filaments and then fitting appropriate filaments to circles. We have shown that in principle it is possible to stabilize an axisymmetric scroll ring with parametric forcing. However, without some method of feedback control this is difficult in practice.

We verified that the unforced twisted scroll ring is a relative equilibrium of the reaction-diffusion equations (there is a frame of reference in which it is a steady state) and that apart from a periodic symmetry resulting from periodic boundary conditions in the drift direction, the twisted scroll ring has no spatial symmetries. We have argued on the basis of our numerical experiments that the dynamics of a twisted scroll ring are not those of a generic relative equilibrium in an $\mathbb{E}(3)$ equivariant system [29,37]. Rather, the dynamics are governed by the symmetry $\mathbb{S E}(2) \times \mathbb{R}$. The basis of the argument is that a twisted scroll ring has a central filament that extends to infinity along some direction and that rotations about axes perpendicular to this direction act discontinuously on the solution fields. Therefore the dynamics of the system will not generate such rotations. We have found that there is bistability between two very slightly different twisted-scroll ring states. Finally we have carefully investigated twisted scroll rings under resonant and near resonant periodic forcing and have shown that its behavior is exactly as expected on the basis of a dynamical systems description, namely resonant drift occurs in a direction perpendicular to the central filament and drift along the central filament is modified.

We have given considerable thought to how one might provide a definitive numerical demonstration of our conjecture that the relevant symmetry of 
the twisted scroll ring is not $\mathbb{E}(3)$. Increasing the size of the simulation volume while either keeping periodic boundary conditions or imposing Neumann boundary conditions in the direction of the central filament would not seem to provide a more definitive result than what we have obtained thus far. So long as the domain is not rotationally symmetric it would not be clear from numerical solutions that the boundary is not selecting a preferred direction. Perhaps simulations in a sufficiently large spherical domain that co-moves with the structure would be adequate, but this would be difficult to program and outside our current focus. Hence we leave it as a conjecture that the appropriate symmetry group for the dynamics of the twisted scroll ring is $\mathbb{S E}(2) \times \mathbb{R}$.

The periodic forcing we have considered here is essentially equivalent to the dynamics near a Hopf bifurcation. The locus of Hopf bifurcations from rigid spiral rotation and to spiral meander in two dimensions is known [32] for the reaction-diffusion equations (1-2). It would be of some interest consider the Hopf bifurcation for the axisymmetric and twisted scroll rings and in particular to establish where the instability occurs in parameter space.

Finally, our approach to understanding waves in three-dimensional excitable media is very different from the approach of filament dynamics. The description of three dimensional structures in terms of filament dynamics requires the "local geometry hypothesis" that filaments move according to their local curvature and twist which are generally assumed to be small [14-17]. These assumptions do not hold for most compact structures such as rings, links and knots, apparently because these structures exhibit substantial non-local filament interaction[10,11]. On the other hand, the dynamical systems approach does not preclude filament interaction, but instead relies only on the basic symmetry properties of solutions. We hope to study other organizing centers (linked rings and knots) with this viewpoint in the future.

\section{Acknowledgement}

We wish to thank P. Ashwin, B. Fiedler, I. Melbourne, B. Sandstede, and C. Wulff for many valuable discussions. DB wishes to acknowledge the hospitality of the Institute for Mathematics and Its Applications, University of Minnesota, where portions of this work were carried out.

Initial conditions for three-dimensional waves

Here we describe the method used for generating initial conditions. This is essentially the method pioneered by Winfree et al. ${ }^{1}$ which derives from a

$\overline{1}$ The method is due to D. Epstein, A. Winfree and T. Poston, unpublished in the 
standard method of embedding an algebraic knot in 3-space [41]. Think of a scroll wave as a stack of two-dimensional slices containing spirals. Then one can generate scroll initial conditions via two mappings: a mapping $\phi$ generating two-dimensional spirals with a known tip location, and a mapping $f$ that stacks the spirals in the desired three-dimensional structure. The mapping $\phi$ is of the form $\phi: \mathbb{R}^{2} \rightarrow \mathbb{R}^{k}$, where $k$ is the number of fields. In our case $k=2$, that is $\phi$ gives the two field values $(u, v)$ at each point in the plane. The stacking map $f: \mathbb{R}^{3} \rightarrow \mathbb{R}^{2}$ send points in three space to the two-dimensional slices. The composition $\phi \circ f$ thus assigns $(u, v)$ values to each point in three space, i.e. generates the initial conditions.

\section{.1 Two-dimensional spirals}

A simple initial condition for spiral waves is a sector of excitation. Expressed in polar coordinates $(r, \theta)$, the excited region can be taken as lying between a wave front at $\theta=\theta_{0}$ and a wave back at $\theta=0$. For kinetics (2), excited regions correspond to $u=1$ and refractory regions to $u=0$. Thus we take $u$ to be initially piecewise constant with $u=1$ for $0 \leq \theta<\theta_{0}$, and $u=0$ otherwise. We take $v$ to be initially piecewise linear with $v=v_{\text {min }} \simeq 0$ at the wave front and $v=v_{\max }$ at the wave back. Thus we have the following expression for $\phi$ :

$$
\left(\begin{array}{c}
r \\
\theta
\end{array}\right) \stackrel{\phi}{\rightarrow}\left\{\begin{array}{l}
\left(\begin{array}{c}
1 \\
v_{\max } \frac{\theta_{0}-\theta}{\theta_{0}}
\end{array}\right) \text { if } 0 \leq \theta<\theta_{0} \\
\left(\begin{array}{c}
0 \\
v_{\max } \frac{\theta_{0}-\theta}{\theta_{0}-2 \pi}
\end{array}\right) \text { if } \theta_{0} \leq \theta<2 \pi
\end{array}\right.
$$

where we have set $v_{\text {min }}=0$. In practice we use $v_{\text {max }}=a$ and $\theta_{0} \approx \pi$.

\section{.2 Complex polynomial maps}

For simplicity assume that $\phi$ generates a spiral whose tip is at the origin in $\mathbb{R}^{2}$. (For the preceding $\phi$ this is approximately true). Then the set of points in $\mathbb{R}^{3}$ that are mapped to the origin by the stacking map $f$ comprise the filament. It is easy to find a continuous mapping that gives rise to a single straight scroll wave. However, as soon as filaments are joined to form rings, finding continuous maps with the appropriate kernel becomes more difficult.

Interesting filaments geometries can be generated from complex polynomial maps. Given a non-constant polynomial in two complex variables $p\left(z_{1}, z_{2}\right)$, the

original form. See $[13,40,11]$ 
equation $p\left(z_{1}, z_{2}\right)=0$ describes a hyper-surface in $\mathbb{C}^{2}$. Denote this by $V$. Now look at the intersection of $V$ with a small 3 -sphere $\mathbb{S}_{\varepsilon}$ centered about some point $q$ on $V$ and define $K=V \cap \mathbb{S}_{\varepsilon}$. As $V$ is a two-dimensional manifold intersecting $\mathbb{S}_{\varepsilon}$ transversely, the real dimension of $K$ must be one. In the simplest case $K$ is a circle embedded into the 3 -sphere $\mathbb{S}_{\varepsilon}$. However, if $q$ is a critical point of the polynomial, then $K$ need not be topologically a circle, and if $K$ is topologically a circle it can be embedded in knotted way. This provides a method for obtaining the stacking map $f$ itself as the composition of two mappings: $f=p \circ s$, where $s$ maps $\mathbb{R}^{3}$ to a three-sphere in $\mathbb{R}^{4}$, and $p$ is the polynomial mapping taking points in $\mathbb{C}^{2} \cong \mathbb{R}^{4}$ to $\mathbb{C}$. The points in $\mathbb{R}^{3}$ that get mapped to the origin in $\mathbb{C}$ under the composition can be chosen via $p$ to be some desired curve or curves.

The standard mapping from $\mathbb{R}^{3}$ into $\mathbb{S}_{\varepsilon}^{3}$ is the stereo-graphic map:

$$
\left(\begin{array}{c}
x \\
y \\
z
\end{array}\right) \stackrel{s}{\rightarrow} \frac{1}{R^{2}+\varepsilon^{2}}\left(\begin{array}{c}
2 \varepsilon^{2} x \\
2 \varepsilon^{2} y \\
2 \varepsilon^{2} z \\
\left(R^{2}-\varepsilon^{2}\right) \varepsilon
\end{array}\right) \cong \frac{1}{R^{2}+\varepsilon^{2}}\left(\begin{array}{c}
2 \varepsilon^{2} x+\mathrm{i} 2 \varepsilon^{2} y \\
2 \varepsilon^{2} z+\mathrm{i}\left(R^{2}-\varepsilon^{2}\right) \varepsilon
\end{array}\right)
$$

where $R^{2} \equiv x^{2}+y^{2}+z^{2}$. Points inside $\mathbb{S}_{\varepsilon}^{2} \subset \mathbb{R}^{3}$ are mapped to the lower hemisphere, points outside $\mathbb{S}_{\varepsilon}^{2}$ to the upper hemisphere of $\mathbb{S}_{\varepsilon}^{3}$.

In numerical simulations we are only interested in a subset of $\mathbb{R}^{3}$, usually a cube, and it is necessary to scale the simulation volume such that under the mapping (.2) this volume covers a sizable portion of the sphere. Scaling the volume to the cube $[-2 \varepsilon, 2 \varepsilon]^{3}$ will cover over $70 \%$ of $\mathbb{S}_{\varepsilon}^{3}$, which is a good choice for most initial conditions. For the twisted scroll ring, it is necessary to scale the $z$-direction differently. Ideally the $z$ coordinate of the simulation cube should be scaled to $[-\infty,-\infty]$ and this can be accomplished via the tan function.

By appropriate choice of the polynomial map we can take $\varepsilon=1 / 2$ in the definition of $s$. Moreover, as we are only interested in a subset of $\mathbb{R}^{3}$, it is possible to use a simpler mapping by dropping the pre-factor in (.2) and using

$$
\left(\begin{array}{l}
x \\
y \\
z
\end{array}\right) \stackrel{s}{\rightarrow}\left(\begin{array}{c}
x \\
y \\
z \\
\left(R^{2}-\frac{1}{4}\right)
\end{array}\right) \cong\left(\begin{array}{c}
x+\mathrm{i} y \\
z+\mathrm{i}\left(R^{2}-\frac{1}{4}\right)
\end{array}\right)
$$

This describes a parabolic surface that touches the sphere $\mathbb{S}_{1 / 4}^{3}$ at $(0,0,0,-1 / 4)$.

In summary, we generate our initial conditions by scaling the simulation volume to the cube $[-1,1]^{3}$, or $[-1,1]^{2} \times(-\infty,-\infty)$ for the twisted scroll ring, 
and then apply the following composition of mappings:

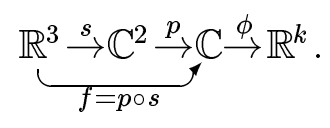

where $s$ is given by $(.3), p$ is chosen according to the desired filament structure, and $\phi$ is given by (.1).

\section{References}

[1] A. T. Winfree. Spiral waves of chemical activity. Science, 175:634-636, 1972.

[2] B.J. Welsh, J. Gomatam, and A.E. Burgess. 3-dimensional chemical waves in the belousov-zhabotinskii reaction. Nature, 304(5927):611-614, 1983.

[3] W. Jahnke, C. Henze, and A. T. Winfree. Chemical vortex dynamics in 3dimensional excitable media. Nature, 336(6200):662-665, 1988.

[4] A. M. Pertsov, R.R. Aliev, and V.I. Krinsky. 3-dimensional twisted vortices in an excitable chemical medium. Nature, 345(6274):419-421, 1990.

[5] D. Stock and S. C. Müller. 3-dimensional reconstruction of scroll waves in the belousov- zhabotinsky reaction using optical tomography. Physica D, 96(14):396-403, 1996.

[6] M. Vinson, S. Mironov, S. Mulvey, and A. Pertsov. Control of spatial orientation and lifetime of scroll rings in excitable media. Nature, 386(6624):477-480, 1997.

[7] A. T. Winfree and S. H. Strogatz. Singular filaments organize chemical waves in 3 dimensions. 2. twisted waves. Physica D, 9(1-2):65-80, 1983.

[8] A. T. Winfree and S. H. Strogatz. Singular filaments organize chemical waves in 3 dimensions. 3. knotted waves. Physica D, 9(3):333-345, 1983.

[9] A. T. Winfree and S. H. Strogatz. Singular filaments organize chemical waves in 3 dimensions. 4. wave taxonomy. Physica D, 13(1-2):221-233, 1984.

[10] A. T. Winfree. Stable particle-like solutions to the nonlinear-wave equations of 3- dimensional excitable media. Siam Review, 32(1):1-53, 1990.

[11] A. T. Winfree. Persistent tangles of vortex rings in excitable media. Physica $D, 84(1-2): 126-147,1995$.

[12] A. T. Winfree. Lingering mysteries about organizing centers in the beluzovzhabotinsky medium and its oregonator model. In R. Kapral and K. Showalter, editors, Chemical Waves and Patterns, pages 3-56. Kluwer, 1995. 
[13] A. T. Winfree. When time breaks down. Princeton Univ. Press, Princeton,NJ, 1987.

[14] A. V. Panfilov, A. N. Rudenko, and A. M. Pertsov. Twisted scroll waves in active 3-dimensional media. Doklady Akademii Nauk Sssr, 279(4):1000-1002, 1984 .

[15] J. P. Keener. The dynamics of 3-dimensional scroll waves in excitable media. Physica D, 31(2):269-276, 1988.

[16] V. N. Biktashev. Diffusion of autowaves - evolution equation for slowly varying autowaves. Physica D, 40(1):83-90, 1989.

[17] V. N. Biktashev, A. V. Holden, and H. Zhang. Tension of organizing filaments of scroll waves. Phil. Trans. R. Soc. Lond. A, 347(1685):611-630, 1994.

[18] K. I. Agladze, V. A. Davydov, and A. S. Mikhailov. Observation of a helicalwave resonance in an excitable distributed medium. JETP Letters, 45(12):767$770,1987$.

[19] V. A. Davydov, V. S. Zykov, and A. S. Mikhailov. Kinematics of autowave patterns in excitable media. Uspekhi Fizicheskikh Nauk, 161(8):45-86, 1991. English in Sov. Phys. Usp. 34,665.

[20] V. N. Biktashev and A. V. Holden. Resonant drift of an autowave vortex in a bounded medium. Physics Letters A, 181(3):216-224, 1993.

[21] O. Steinbock, V. Zykov, and S. C. Müller. Control of spiral-wave dynamics in active media by periodic modulation of excitability. Nature, 366(6453):322-324, 1993.

[22] V. N. Biktashev and A. V. Holden. Design principles of a low-voltage cardiac defibrillator based on the effect of feedback resonant drift. Journal of Theoretical Biology, 169(2):101-112, 1994.

[23] M. Braune, A. Schrader, and H. Engel. Entrainment and resonance of spiral waves in active media with periodically modulated excitability. Chemical Physics Letters, 222(4):358-362, 1994.

[24] V. Zykov, O. Steinbock, and S. C. Müller. External forcing of spiral waves. Chaos, 4(3):509-518, 1994.

[25] V. N. Biktashev and A. V. Holden. Resonant drift of autowave vortices in 2 dimensions and the effects of boundaries and inhomogeneities. Chaos Solitons \& Fractals, 5(3-4):575-622, 1995.

[26] V. N. Biktashev and A. V. Holden. Control of reentrant activity in a model of mammalian atrial tissue. Proceedings of the Royal Society of London Series B - Biological Sciences, 260(1358):211-217, 1995.

[27] A. Schrader, M. Braune, and H. Engel. Dynamics of spiral waves in excitable media subjected to external periodic forcing. Physical Review E, 52(1 PtA):98$108,1995$. 
[28] R. M. Mantel and D. Barkley. Periodic forcing of spiral waves in excitable media. Phys. Rev. E, 54:4791-4802, 1996.

[29] B. Sandstede, A. Scheel, and C. Wulff. Bifurcations and dynamics of spiral waves. J. Nonl. Science, to appear, 1998.

[30] D. Barkley. A model for fast computer-simulation of waves in excitable media. Physica D, 49(1-2):61-70, 1991.

[31] M. Dowle, R. M. Mantel, and D. Barkley. Fast simulations of waves in threedimensional excitable media. Int. J. Bifur. Chaos, 7(11):2529-2546, 1997.

[32] D. Barkley. Euclidean symmetry and the dynamics of rotating spiral waves. Physical Review Letters, 72(1):164-167, 1994.

[33] M. J. Cohen. Fitting a circle to data points. newsgroup: sci.math, aug 1996.

[34] A.V. Panfilov and A.M. Pertsov. Vortex ring in 3-dimensional active medium described by reaction diffusion-equations. DOKLADY AKADEMII NAUK SSSR, 274(6):1500-1503, 1984.

[35] A. V. Panfilov, A. N. Rudenko, and V. I. Krinsky. Turbulent rings in 3dimensional active media with diffusion by 2 components. Biofizika, 31(5):850$854,1986$.

[36] D. Barkley. Spiral meandering. In R. Kapral and K. Showalter, editors, Chemical Waves and Patterns, pages 163-190. Kluwer, 1995.

[37] P. Ashwin and I. Melbourne. Noncompact drift for relative equilibria and relative periodic orbits. Nonlinearity, 10:595-610, 1997.

[38] B. Fiedler, B. Sandstede, A. Scheel, and C. Wulff. Bifurcation from relative equilibria of noncompact group actions: skew products, meanders, and drifts. Documenta Mathematica, 1:479-505, 1996.

[39] B. Sandstede, A. Scheel, and C. Wulff. Dynamics of spiral waves on unbounded domains using center-manifold reductions. J. Diff. Eq., 141:122 -149, 1997.

[40] C. Henze and A. T. Winfree. The differential geometry of scroll waves. Int. J. Bifur. Chaos, 1(4):723-744, 1991.

[41] K. Brauner. Zur Geometrie der Funktionen zweier komplexer Veränderlicher iii,iv. Abh. Math. Sem. Hamburg, 6:8-54, 1928. 